\title{
2 PROBLEMAS DOS CUIDADORES DE DOENTES COM ESQUIZOFRENIA: A SOBRECARGA FAMILIAR
}

\author{
| Joana Morgado Alves'; Assunção Laranjeira Almeida²; Maria Augusta Pereira da Mata ${ }^{3}$; Maria Helena Pimentel ${ }^{4}$ |
}

\section{RESUMO}

CONTEXTO: A esquizofrenia é uma doença mental grave. Pelas suas características leva a um aumento tanto da sobrecarga objetiva como subjetiva dos familiares da pessoa que a suporta.

OBJETIVO: Avaliar os problemas dos cuidadores de doentes com esquizofrenia em termos de sobrecarga objetiva e subjetiva, ajuda recebida, atitudes positivas e criticismo, bem como a sua relação com as variáveis de caracterização sociodemográfica e anos de contacto do cuidador com o doente. MÉTODOS: Estudo quantitativo, transversal, descritivo correlacional numa amostra não probabilística, por conveniência, constituída por 35 familiares de doentes com o diagnóstico de esquizofrenia. Recolha de dados de junho a setembro de 2016 a familiares na consulta externa ou hospital de dia do Departamento de Psiquiatria e Saúde Mental dum hospital da Zona Centro de Portugal. Instrumento de recolha com questões de caracterização sociodemográfica, anos de contacto enquanto cuidador e Questionário de Problemas Familiares (FPQ) na sua versão original de Morosini et al., (1991), validado para a população portuguesa por Xavier et al., (2002).

RESULTADOS: Amostra constituída maioritariamente por familiares do género feminino (54,3\%), casados (68,6\%), grau de parentesco pai/mãe (60\%), habilitações literárias $2^{\circ}$ ciclo (40\%), empregados (40\%) e contato com o doente após diagnóstico de esquizofrenia há mais de 10 anos (57,1\%). No FPQ a média mais elevada foi obtida na sobrecarga subjetiva $(M=2,35)$ com diferenças estatisticamente significativas segundo o grau de escolaridade $(\mathrm{KW}=6,747 ; \mathrm{p}=0,034)$, seguida de perto pelas atitudes positivas $(\mathrm{M}=2,34)$ e ajuda recebida $(\mathrm{M}=2,33)$.

CONCLUSÃO: Existe sobrecarga familiar na amostra em estudo. $\mathrm{O}$ apoio dos enfermeiros reflete-se na ajuda recebida. Assim, nas intervenções que reduzam a sobrecarga familiar o enfermeiro especialista em sáude mental dos cuidados de saúde primários tem um papel primordial.

\section{PALAVRAS-CHAVE: Burnout; Cuidadores; Esquizofrenia; Relações familiares}

\section{RESUMEN}

\section{"Problemas de los cuidadores de pacientes con esquizofrenia: La sobrecarga familiar" \\ CONTEXTO: La esquizofrenia es una enfermedad mental grave. Debido a} sus características conduce a un aumento tanto de la sobrecarga objetiva como subjetiva en los familiares de la persona que la soporta .

OBJETIVO: Evaluar los problemas de los cuidadores de pacientes con esquizofrenia en términos de sobrecarga objetiva y subjetiva, ayuda recibida, actitudes positivas y criticismo, así como su relación con las variables de caracterización sociodemográfica y años de contacto del cuidador con el enfermo. MÉTODOS: Estudio cuantitativo, descriptivo, correlacional, transversal, una muestra no aleatoria, por conveniencia, que consiste en 35 miembros de la familia de los pacientes con el diagnostico de esquizofrenia. La recogida de datos se llevó a cabo entre junio y septiembre de 2016 para la familia en el ambulatorio o de hospital de día del Departamento de Psiquiatría y Salud Mental CHBV. El instrumento de recolección de datos se compone de temas de caracterización socio-demográficos, años de contacto y Cuestionario de Problemas de la familia (PPQ) en su versión original de Morosini et al., (1991), validado para la población portuguesa por Xavier et al., (2002).

RESULTADOS: La muestra que consiste principalmente de mujeres de la familia (54,3\%), que están casados (68,6\%), relación padre / madre $(60 \%)$, con titulación de $2^{\circ}$ ciclo (40\%), empleados (40\%) y tienen contacto con el paciente después del diagnóstico de la esquizofrenia durante más de 10 años (57,1\%). En PPQ se obtuvo el promedio más alto de carga subjetiva $(M=2,35)$ con diferencias estadísticamente significativas según el nivel de la escolarización $(\mathrm{KW}=6,747 ; \mathrm{p}=0,034)$, seguido de cerca por una actitud positiva $(\mathrm{M}=2.34)$ y la asistencia recibida $(\mathrm{M}=2,33)$.

CONCLUSIÓN: Los resultados indican que hay carga familiar en la muestra en estudio. El apoyo de las enfermeras se refleja en la ayuda recibida. Por lo tanto, en las intervenciones que reducen la carga familiar, la enfermera especialista en salud mental de la atención primaria tiene un papel clave.

\section{DESCRIPTORES: Burnout; Cuidadores; Esquizofrenia; Rela-} ciones familiares

\section{ABSTRACT \\ "Problems of caregivers of patients with schizophrenia: The family burden" \\ BACKGROUND: Schizophrenia is a serious mental disease. By its characteris-} tics it leads to an increase of both objective and subjective burden of relatives. AIM: To assess the problems of caregivers of patients with schizophrenia in terms of objective and subjective burden, received help, positive atitudes and criticism as well as their connection with social and demographic characterization variables and caregiver's contact time with the patient.

METHODS: Quantitative, cross-sectional, descriptive and correlational study with a convenient non-random sample of 35 family members of patients diagnosed with schizophrenia. Data collection was carried out with family members at the consultation or Day Hospital of Psychiatry and Mental Health Department at a Hospital of the Central Zone of Portugal from June to September 2016. Data collection tool included social and demographic questions, contact years with the patient and the original version of Family Problems Questionnaire (FPQ) by Morosini et al., (1991), validated to the Portuguese population by Xavier et al., (2002).

RESULTS: The sample consisted mainly of female family members $(54,3 \%)$, married $(68,6 \%)$, patient's father/mother $(60 \%)$, holding 5 years of schooling (2nd cycle of basic scholarship) $40 \%$ ), employed (40\%) and with 10 or more years of contact with the patient $(57,1 \%)$. The highest average in FPQ was obtained in subjective overload $(M=2,35)$ with statistically significant differences according to scholarship level $(\mathrm{KW}=6,747 ; \mathrm{p}=0,034)$, followed by the positive attitudes $(M=2,34)$ and received help $(M=2,33)$.

CONCLUSION: There is family burden in the studied sample. Nurses' support is reflected on the received help. Thus, specialist nurse in mental health within primary health care performs a major role in the interventions aiming at the reduction of family overload.

\section{KEYWORDS: Burnout; Caregivers; Schizophrenia; Family relationships}

Submetido em 30-03-2017

Aceite em 22-11-2017

1 Mestre; Enfermeira no Centro Hospitalar do Baixo Vouga, Departamento de Psiquiatria e Saúde Mental, 3810-501 Aveiro, Portugal, joanaalves83@hotmail.com

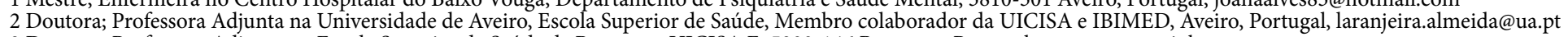

3 Doutora; Professora Adjunta na Escola Superior de Saúde de Bragança, UICISA:E, 5300-146 Bragança, Portugal, augustamata@ipb.pt

4 Doutora; Professora Coordenadora na Escola Superior de Saúde de Bragança, UICISA:E, Avenida D. Afonso V, $5300-146$ Bragança, Portugal, hpimentel@ipb.pt

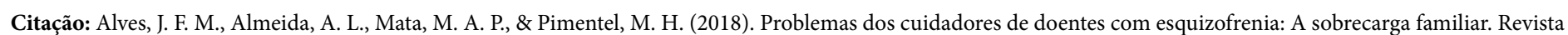
Portuguesa de Enfermagem de Saúde Mental (19), 08-16. doi: 10.19131/rpesm.0197 


\section{INTRODUÇÃO}

O diagnóstico de Esquizofrenia sofreu alterações significativas na versão do DSM-V, 2014). Abandonou a divisão da esquizofrenia em subtipos. $O$ critério que define a sintomatologia característica (Critério A) continua a requerer a presença, no mínimo, de dois dos cinco sintomas mas a atual versão exige que pelo menos um deles seja positivo.

Carateriza-se por uma perturbação mental grave que apresenta curso e prognóstico variáveis. A sintomatologia engloba a presença de disfunções cognitivas, emocionais e comportamentais que alteram a perceção, o raciocínio lógico, a linguagem e a comunicação, o afeto, a fluência, conteúdo do pensamento e do discurso, a capacidade hedónica, a vontade, o impulso e a atenção. Este conjunto de sinais e sintomas surge associado a uma disfunção social e ocupacional (American Psychiatric Association, 2013), habitualmente com recorrentes recaídas, com sintomas residuais e recuperação social muito deficiente. Parte da gravidade da doença diz respeito, precisamente, ao facto de raramente o doente conseguir voltar ao funcionamento pré-mórbido (Silva, 2006).

Em 2001 a Organização Mundial de Saúde, no Relatório Mundial de Saúde dedicado à saúde mental, definiu metas para o início da desinstitucionalização e criação de serviços na comunidade com cuidados individualizados para doentes mentais graves. Na Declaração Europeia de Saúde Mental (Helsínquia, 2005), os Ministros da Saúde da União Europeia comprometeram-se a assegurar cuidados aos doentes mentais graves a partir da comunidade (Resolução do Conselho de Ministros, 2008). Em conformidade, o Plano Nacional de Saúde Mental de 2007-2016 reconhece as perturbações psiquiátricas e os problemas de saúde mental como a principal causa de incapacidade e uma das principais causas de morbilidade, nas sociedades atuais, traçando como prioridade o desenvolvimento de equipas de saúde mental comunitária. Esta prioridade é ainda mais relevante se considerarmos que $22,9 \%$ da população portuguesa tem perturbações psiquiátricas e 14,3\% recebe tratamentos em saúde mental (Caldas de Almeida et al., 2013).

Com efeito, desde que a desinstitucionalização psiquiátrica se tornou emergente a inserção efetiva da família no novo paradigma de assistência em saúde mental ganhou acuidade.
No entanto, é necessário que os profissionais de saúde estejam atentos à saúde mental de todos os membros da família para que a desinstitucionalização psiquiátrica não passe a ter um efeito negativo, contrário ao esperado (Borba, Schwartz \& Kantorski, 2008; Gomes, 2015; Stephanie, 2012). A desinstitucionalização só pode ser bem-sucedida se os doentes encontrarem na coletividade recursos organizados que promovam suporte e orientação.

Vários estudos comprovam a sobrecarga familiar perante doentes mentais graves (Borba et al., 2008; Pinho e Pereira, 2015; Stephanie, 2012; Tenório de Albuquerque, Oliveira Cintra e Bandeira, 2010; Xavier, Pereira, Corrêa e Caldas de Almeida, 2002).

A sobrecarga familiar segundo Tenório de Albuquerque et al., (2010) e Xavier et al., (2002), pode dividir-se fundamentalmente em duas componentes: sobrecarga objetiva e sobrecarga subjetiva. A sobrecarga objetiva diz respeito ao impacto direto das modificações e limitações determinadas pela doença de um indivíduo nos seus familiares. Já a sobrecarga subjetiva relaciona-se com o conjunto de sentimentos decorrente da vivência intrapsíquica destas limitações, refletiva em termos de impacto psicológico.

Por sua vez, Borba et al., (2008) classificam a sobrecarga familiar como sobrecarga física e emocional, sobrecarga do cuidado e sobrecarga financeira. A sobrecarga física e emocional identifica-se pelo aparecimento de doenças relacionadas ao sistema nervoso (como gastrite e problemas gastrointestinais), evidenciando como os problemas de ordem emocional se repercutem na saúde física.

Emocionalmente os familiares podem encontrar-se vulneráveis pela privação do sono ou por não suportarem a convivência. Neste contexto cuidar pode tornarse uma tarefa difícil, quer seja pela falta de apoio e comprometimento dos demais membros da família, quer seja pelas exigências ou solicitações do familiar doente. Em termos de sobrecarga financeira, os mesmos autores, salientam a situação económica desfavorável de muitas famílias, agravando o problema e a necessidade de reorganizar o orçamento familiar. Ainda, prever faltas ao trabalho para acompanhar e atender as necessidades de cuidados do familiar portador de doença mental podendo, não raras vezes, comprometer a única fonte de rendimento da família.

Em Portugal, regra geral, a família constitui-se como rede de suporte natural e muitos doentes vivem com pelo menos um familiar direto. 
É, portanto, natural que a família deva ser incluída nos planos de tratamento e reabilitação (Gomes, 2015; Gonçalves-Pereira, Xavier, Neves, Barahona-Correa \& Fadden 2006).De acordo com Pinho e Pereira (2015: 16) George Brown “( ...)comprovou-se maior percentagem de recaídas nos doentes que viviam em casa de familiares do que naqueles que viviam sozinhos ou com outros doentes".

Assim, o envolvimento ativo da família no processo de tratamento considera-se de extrema importância, pelo que as intervenções educativas poderão constituir-se uma prioridade de ação. A educação em saúde pretende reduzir o stresse familiar, providenciar suporte social e promoção do empoderamento (Gonçalves-Pereira et al., 2006; Lucksted, McFarlane, Downing \& Dixon, 2012; Pfammatter, Andres \& Brenner, 2012). A implementação de intervenções familiares no tratamento de doentes com perturbações psicóticas surge da necessidade de dar resposta efetiva às necessidades da família e minimizar as dificuldades, assente na perceção de que a sua integração nos cuidados representa um benefício em termos de prognóstico e de indicadores de bem-estar (Pharoah, Mari, Rathbone \& Wong, 2010). Também o Relatório da Primavera (2015) vem afirmar idênticos propósitos.

Em suma para que a desinstitucionalização tenha êxito é necessário proporcionar aos familiares cuidadores estratégias e ferramentas que minimizem o impacto negativo da doença tornando-os resilientes, capacitando-os e promovendo o seu empoderamento (Almeida, Dimenstein e Severo, 2010; Carvalho, 2012). Ainda, cuidados domiciliários necessários à sua recuperação (Tomás, 2013). Assim, este estudo tem como objetivo avaliar os problemas dos familiares/cuidadores de doentes com esquizofrenia, bem como a sua relação com as variáveis de caracterização sociodemográfica e tempo de contacto com o doente mental.

\section{MÉTODOS}

Realizámos um estudo quantitativo, transversal, descritivo correlacional numa amostra não probabilística, por conveniência, constituída por 35 familiares de doentes com o diagnóstico de esquizofrenia.

Como critérios de inclusão foram considerados: familiares/cuidadores de doente mental com esquizofrenia e com diagnóstico há mais de um ano; com capacidade para ler, compreender e completar com sucesso os instrumentos de colheita de dados.
A recolha de dados ocorreu no período de junho a setembro de 2016, aos familiares que se encontravam a acompanhar o utente na consulta externa ou no hospital de dia do Departamento de Psiquiatria e Saúde Mental do Centro Hospitalar do Baixo Vouga (CHBV, E.P.E).

O instrumento de colheita de dados contempla questões de caracterização sociodemográfica e número de anos de contacto com o doente mental ao qual juntámos o Questionário de Problemas Familiares (FPQ), desenvolvido na sua versão original na língua inglesa pelo Instituto de Saúde Pública de Roma e pelo Departamento de Psiquiatria da Universidade de Nápoles (Morosini, Roncone, \& Casacchia, 1991), validado para a população portuguesa por Xavier et al., (2002). Estruturalmente o FPQ é um instrumento de autopreenchimento constituído por 29 itens avaliados de 1 a 4 , em escala de tipo Likert, composto por cinco subescalas: sobrecarga objetiva, sobrecarga subjetiva, ajuda recebida de profissionais e/ou rede social, atitudes positivas e criticismo. Ainda, por três secções adicionais: sobrecarga em crianças, custos diretos e indiretos (Tabela 1). Apresentou, segundo os autores, na validação para a população portuguesa características psicométricas satisfatórias.

Tabela 1 - FPQ e Suas Subescalas

\begin{tabular}{|l|l|c|}
\hline Fator & Subescalas & Itens \\
\hline $\mathbf{1}$ & Sobrecarga objetiva & $\begin{array}{r}14,15,16,17,18,19, \\
20,21\end{array}$ \\
\hline $\mathbf{2}$ & Sobrecarga subjetiva & $29,30,34,35,37,38,47$ \\
\hline $\mathbf{3}$ & Ajuda recebida & $3^{\star}, 4^{\star}, 5^{\star}, 6^{\star}, 9,10,58$ \\
\hline $\mathbf{4}$ & Atitudes positivas & $48,55,60,62$ \\
\hline $\mathbf{5}$ & Criticismo & $54,63,64^{\star}$ \\
\hline \multirow{2}{*}{$\begin{array}{l}\text { Secções } \\
\text { adicionais }\end{array}$} & Sobrecarga em crianças & 39,41 \\
\cline { 2 - 3 } & Custos diretos & $67,75,83$ \\
\cline { 2 - 3 } & Custos indiretos & 22,23 \\
\hline
\end{tabular}

* Escala inversa

Tendo em conta os objetivos delineados para a presente investigação foram avaliadas somente as subescalas referentes à sobrecarga objetiva, sobrecarga subjetiva, ajuda recebida, atitudes positivas e criticismo não sendo avaliadas as secções adicionais.

Foi obtida autorização à Comissão de Ética do Centro Hospitalar Baixo Vouga, E.P.E. e solicitada autorização ao Conselho de Administração desse Centro Hospitalar. Procedemos ao estudo da consistência interna do FPQ para a presente investigação e obtivemos valores de Alfa de Cronbach de 0,948 para o total dos 29 itens. 
Para a análise estatística utilizámos o nível de significância de $5 \%(\mathrm{p}=0,05)$. Recorremos aos testes não paramétricos de Mann-Whitney e Teste de Kruskal Wallis tendo em conta o reduzido tamanho da amostra e a não distribuição normal das variáveis.

\section{RESULTADOS}

A amostra em estudo é constituída maioritariamente por indivíduos do sexo feminino (54,3\%), casados $(68,6 \%)$ e progenitores (pai e/ou mãe) como grau de parentesco com o doente $(60,0 \%)$. Têm idades compreendidas entre os 17 e os 77 anos, com uma média de idade de 53,1 anos ( $\mathrm{DP}=14,18)$. Quanto à escolaridade e situação ocupacional a maioria tem o $2^{\circ}$ ciclo (40\%) e emprego (40\%). No que diz respeito ao contacto com o doente mental mais de metade $(57,1 \%)$ tem contacto há mais de 10 anos (Tabela 2).

Tabela 2 - Caracterização Sociodemográfica dos Inquiridos $(n=35)$ e Tempo de Contacto com o Doente Mental

\begin{tabular}{|c|c|c|c|}
\hline & & $\mathbf{n}$ & $\%$ \\
\hline \multirow[t]{2}{*}{ Sexo } & Masculino & 14 & 40,0 \\
\hline & Feminino & 19 & 54,3 \\
\hline Idade & Média $=53,1$ anos & \multicolumn{2}{|c|}{$\mathrm{Dp}=14,18$ anos } \\
\hline \multirow[t]{4}{*}{ Estado civil } & Casado & 24 & 68,6 \\
\hline & Solteiro & 4 & 11,4 \\
\hline & Separado/divorciado & 5 & 14,3 \\
\hline & Viúvo & 2 & 5,7 \\
\hline \multirow[t]{4}{*}{ Parentesco c/ doente } & Pai/mãe & 21 & 60,0 \\
\hline & Cônjuge & 6 & 17,1 \\
\hline & Irmão/irmã & 2 & 5,7 \\
\hline & Outros & 6 & 17,1 \\
\hline \multirow[t]{3}{*}{ Grau de escolaridade } & $1^{\circ}$ ciclo & 12 & 34,3 \\
\hline & $2^{\circ}$ ciclo & 14 & 40,0 \\
\hline & Superior & 9 & 25,7 \\
\hline \multirow[t]{4}{*}{ Situação ocupacional } & Empregado & 14 & 40,0 \\
\hline & Desempregado & 5 & 14,3 \\
\hline & Aposentado & 12 & 34,3 \\
\hline & Outro & 4 & 11,4 \\
\hline \multirow[t]{4}{*}{ Contacto c/doente } & 1-3 anos & 9 & 25,7 \\
\hline & 4-6 anos & 2 & 5,7 \\
\hline & 7-10 anos & 4 & 11,4 \\
\hline & $>10$ anos & 20 & 57,1 \\
\hline
\end{tabular}

As análises efetuadas centraram-se no estudo das cinco subescalas do Questionário de Problemas Familiares, conforme Tabela 3. A mais representativa corresponde à sobrecarga subjetiva $(\mathrm{M}=2,35)$, seguida de perto pelas atitudes positivas $(M=2,34)$ e apoio recebido $(M=2,33)$.
Tabela 3 - Subescalas do FPQ Média e Desvio-Padrão

\begin{tabular}{|l|c|c|c|}
\hline Subescalas & $\mathbf{n}^{\circ}$ itens & M & DP \\
\hline Sobrecarga objetiva & 8 & 1,99 & 0,702 \\
\hline Sobrecarga subjetiva & 7 & 2,35 & 0,847 \\
\hline Ajuda recebida & 7 & 2,33 & 0,785 \\
\hline Atitudes positivas & 4 & 2,34 & 0,637 \\
\hline Criticismo & 3 & 1,71 & 0,497 \\
\hline
\end{tabular}

O índice total de sobrecarga objetiva foi de 1,99 $\pm 0,702$, com pontuações mais elevadas nos itens: problemas com o sono $(2,31 \pm 0,900)$; negligenciar outros familiares $(2,28 \pm 1,137)$; limitação dos períodos de lazer $(2,18 \pm 1,103)$; dificuldade em fazer férias $(2,06 \pm 1,027)$. $\mathrm{O}$ valor do índice total de sobrecarga subjetiva foi de $2,35 \pm 0,847$, o mais alto de todas as subescalas avaliadas, os itens mais pontuados são: desapontamento pela evolução do doente $(2,89 \pm 1,051)$; responsabilização do doente $(2,57 \pm 1,267)$; preocupação com o futuro dos familiares $(2,54 \pm 1,094)$.

$\mathrm{Na}$ subescala ajuda recebida o índice global foi de $2,33 \pm 0,785$, com as pontuações mais elevadas a verificarem-se nos itens: ajuda por parte dos enfermeiros $(2,97 \pm 0,918)$; informação dada por profissionais de saúde $(2,58 \pm 1,062)$; emergência/ajuda de profissionais de saúde $(2,53 \pm 1,016)$.

$\mathrm{Na}$ subescala atitudes positivas encontrou-se um índice de 2,34 $\pm 0,637$, com todos os itens a pontuar, em média, em valores muito próximos e superiores a 2 .

Finalmente o criticismo cujo valor global médio $(1,71 \pm 0,497)$ é o mais baixo de todas as subescalas avaliadas, particularmente, nos itens: vontade de se separar o doente $(1,43 \pm 0,739)$ e ideia de que o doente tenta estorvar $(1,47 \pm 0,788)$ (Tabela 4$)$.

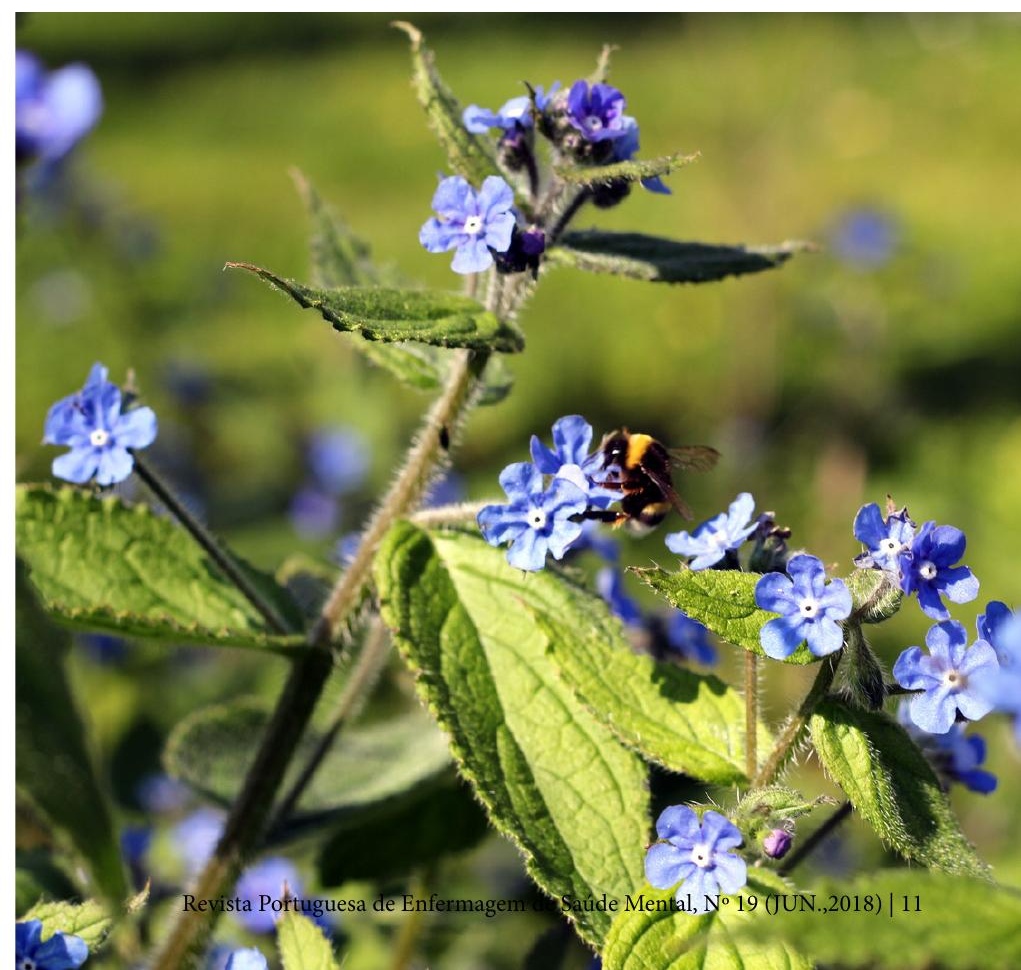


Tabela 4 - Estatísticas Descritivas dos Itens de cada Subescala do FPQ

\begin{tabular}{|c|c|c|c|c|c|c|}
\hline & Sobrecarga Objetiva & $\mathbf{N}$ & Média & Mediana & Moda & DP \\
\hline 14 & Problemas com o sono & 35 & 2,31 & 2 & 2 & 0,900 \\
\hline 15 & Limitação dos períodos de lazer & 33 & 2,18 & 2 & 1 & 1,103 \\
\hline 16 & Dificuldade de sair ao fim-de-semana & 35 & 1,77 & 1 & 1 & 1,087 \\
\hline 17 & Limitação em receber convidados & 35 & 1,77 & 1 & 1 & 1,003 \\
\hline 18 & Restrição da vida social & 35 & 1,86 & 2 & 2 & 0,733 \\
\hline 19 & Problemas de assiduidade no trabalho & 32 & 1,69 & 1,5 & 1 & 0,859 \\
\hline 20 & Negligenciar de outros familiares & 25 & 2,28 & 2 & 1 & 1,137 \\
\hline 21 & Dificuldade em fazer férias & 35 & 2,06 & 2 & 1 & 1,027 \\
\hline \multicolumn{7}{|c|}{ Sobrecarga Subjetiva } \\
\hline 29 & Sensação de esgotamento iminente & 35 & 2,06 & 2 & 1 & 1,027 \\
\hline 30 & Sentimentos de depressão e choro & 35 & 2,37 & 2 & 2 & 0,877 \\
\hline 34 & Preocupação com o futuro dos familiares & 35 & 2,54 & 2 & 2 & 1,094 \\
\hline 35 & Sensação de ser observado (com o doente) & 33 & 2,03 & 2 & 1 & 1,159 \\
\hline 38 & Responsabilização do doente & 35 & 2,57 & 3 & 4 & 1,267 \\
\hline 47 & Desapontamento pela evolução do doente & 35 & 2,89 & 3 & 4 & 1,051 \\
\hline 37 & Culpa por transmissão da doença & 35 & 2,00 & 2 & 1 & 0,939 \\
\hline \multicolumn{7}{|c|}{ Ajuda Recebida } \\
\hline $3^{*}$ & Ajuda de familiares ou amigos & 33 & 2,30 & 2 & 2 & 0,984 \\
\hline $4^{*}$ & Informação dada por profissionais de saúde & 33 & 2,58 & 3 & 2 & 1,062 \\
\hline $5^{*}$ & Emergência, ajuda de família/amigos & 33 & 2,45 & 2 & 2 & 0,938 \\
\hline $6^{*}$ & Emergência,ajuda de profissionais de saúde & 32 & 2,53 & 3 & 3 & 1,016 \\
\hline 9 & Ajuda por parte dos médicos & 33 & 2,48 & 3 & 3 & 1,064 \\
\hline 10 & Ajuda por parte dos enfermeiros & 33 & 2,97 & 3 & 3 & 0,918 \\
\hline 58 & Ajuda por parte de outras entidades & 35 & 1,51 & 1 & 1 & 0,887 \\
\hline \multicolumn{7}{|c|}{ Atitudes Positivas } \\
\hline 48 & Satisfação com a ajuda dada pelo doente & 29 & 2,03 & 2 & 2 & 0,865 \\
\hline 55 & Satisfação pela cooperação do doente & 35 & 2,43 & 2 & 2 & 1,119 \\
\hline 60 & Elogio da sensibilidade do doente & 35 & 2,43 & 2 & 2 & 0,884 \\
\hline 62 & Elogio de qualidades do doente & 32 & 2,44 & 2 & 2 & 1,076 \\
\hline \multicolumn{7}{|c|}{ Criticismo } \\
\hline 54 & Ideia de que o doente tenta estorvar & 34 & 1,47 & 1 & 1 & 0,788 \\
\hline 63 & Vontade de se separar do doente & 35 & 1,43 & 1 & 1 & 0,739 \\
\hline $64^{*}$ & Extrair algo de positivo da situação & 34 & 2,24 & 2 & 2 & 0,923 \\
\hline
\end{tabular}

Nota: os resultados de cada iten variam entre 1 e 4 . 
No Quadro 1 estão representados os valores médios das cinco subescalas do FPQ segundo as variáveis de caracterização dos inquiridos e o tempo de contacto com o doente mental.

Em relação à sobrecarga objetiva os cuidadores do sexo feminino $(2,09)$, com idades iguais ou superiores a 65 anos $(1,89)$, viúvos $(1,82)$, irmãos do doente $(2,24)$, com o $1^{\circ}$ ciclo de formação literária $(2,21)$ e cuidadores há mais de dez anos $(2,14)$ são os que evidenciam médias mais elevadas. No entanto, a aplicação dos testes U de Mann-Whitney e de Kruskal-Wallis não confirmou a existência de diferenças estatisticamente significativas entre os grupos.

Quadro 1 - Valores Médios das Cinco Subscalas do FPQ Segundo as Variáveis de Caraterização e Tempo de Contacto com o Doente

\begin{tabular}{|c|c|c|c|c|c|c|c|c|c|c|c|}
\hline & & \multicolumn{2}{|c|}{ Sob. Objetiva } & \multicolumn{2}{|c|}{ Sob. Subjetiva } & \multicolumn{2}{|c|}{ Ajuda Recebida } & \multicolumn{2}{|c|}{ Atit. Positivas } & \multicolumn{2}{|c|}{ Criticismo } \\
\hline & & ${ }^{-} \mathrm{X}$ & $\mathbf{p}$ & $-\mathrm{X}$ & $\mathbf{p}$ & ${ }^{-} \mathrm{X}$ & $\mathbf{p}$ & ${ }^{-} \mathrm{X}$ & $\mathbf{p}$ & $-\mathrm{X}$ & $\mathbf{p}$ \\
\hline \multirow[t]{2}{*}{ Sexo } & Masculino & 1,84 & \multirow{2}{*}{$0,418^{\star}$} & 2,36 & \multirow{2}{*}{$0,788^{*}$} & 2,33 & \multirow{2}{*}{$1,000^{*}$} & 2,18 & \multirow{2}{*}{$0,212^{*}$} & 1,50 & \multirow{2}{*}{$0,123^{x}$} \\
\hline & Feminino & 2,09 & & 2,48 & & 2,31 & & 2,43 & & 1,79 & \\
\hline \multirow[t]{2}{*}{ G. Etário } & $<65$ anos & 1,89 & \multirow{2}{*}{$0,077^{\star}$} & 2,29 & \multirow{2}{*}{$0,084^{*}$} & 2,91 & \multirow{2}{*}{$0,984^{\star}$} & 2,30 & \multirow{2}{*}{$0,537^{\star}$} & 1,62 & \multirow{2}{*}{$0,141^{*}$} \\
\hline & $\geq 65$ anos & 2,36 & & 2,28 & & 2,78 & & 2,48 & & 1,88 & \\
\hline \multirow[t]{4}{*}{ Estado civil } & Casado & 1,86 & \multirow{4}{*}{$0,187^{1}$} & 2,47 & \multirow{4}{*}{$0,131^{1}$} & 2,32 & \multirow{4}{*}{$0,638^{1}$} & $0,537^{\star}$ & \multirow{4}{*}{$0,632^{1}$} & 1,69 & \multirow{4}{*}{$0,054^{1}$} \\
\hline & Solteiro & 2,67 & & 2,21 & & 2,52 & & 2,17 & & 1,25 & \\
\hline & Sep/divor & 1,82 & & 1,88 & & 2,22 & & 2,67 & & 1,60 & \\
\hline & Viúvo & 3,11 & & 3,42 & & 1,67 & & 2,79 & & 2,33 & \\
\hline \multirow[t]{4}{*}{ Parentesco c/ doente } & $\mathrm{Pai} / \mathrm{mãe}$ & 1,93 & \multirow{4}{*}{$0,813^{1}$} & 2,39 & \multirow{4}{*}{$0,709^{1}$} & 2,30 & \multirow{4}{*}{$0,844^{1}$} & 2,49 & \multirow{4}{*}{$0,099^{1}$} & 1,78 & \multirow{4}{*}{$0,361^{1}$} \\
\hline & Cônjuge & 2,09 & & 2,42 & & 2,52 & & 2,39 & & 1,61 & \\
\hline & Irmão/irmã & 2,24 & & 3,00 & & 2,00 & & 1,67 & & 1,33 & \\
\hline & Outros & 1,95 & & 2,28 & & 2,13 & & 1,94 & & 1,44 & \\
\hline \multirow[t]{3}{*}{ Escolaridade } & $1^{\circ}$ ciclo & 2,21 & \multirow{3}{*}{$2,59^{1}$} & 2,92 & & 2,27 & & 2,58 & & 1,92 & \\
\hline & $2^{\circ}$ ciclo & 1,99 & & 2,23 & $0,034^{1}$ & 2,23 & $0,782^{1}$ & 2,26 & $0,431^{1}$ & 1,59 & $0,094^{1}$ \\
\hline & Superior & 1,65 & & 2.02 & & 2.42 & & 2.20 & & 1,44 & \\
\hline Situação ocupacional & Empregado & 1,74 & & 2,00 & & 2,45 & & 2,37 & & 1,70 & \\
\hline & Desemprego & 2,15 & 01001 & 2,53 & 01021 & 1,87 & 05411 & 2,33 & 07241 & 1,70 & $0 \circ 0_{1}$ \\
\hline & Aposentado & 2,11 & $0,18 y^{1}$ & 2,71 & $0,102^{2}$ & 2,36 & $0,541^{1}$ & 2,22 & $0, / 24$ & 1,67 & $0,80 /$ \\
\hline & Outro & 2,25 & & 2,79 & & 2,07 & & 2,69 & & 1,50 & \\
\hline Tempo de contacto c/ & $1-3$ anos & 1,83 & & 2,20 & & 2,17 & & 2,31 & & 1,78 & \\
\hline & 4-6 anos & 1,64 & $0509^{1}$ & 1,92 & $0359^{1}$ & 3,50 & $0235^{1}$ & 2,63 & $0898^{1}$ & 1,58 & $0744^{1}$ \\
\hline & 7-10 anos & 1,69 & & 3,04 & & 2,00 & & 2,44 & & 1,50 & \\
\hline & $>10$ anos & 2,14 & & 2,43 & & 2,29 & & 2,29 & & 1,66 & \\
\hline
\end{tabular}

${ }^{1}$ Teste de Kruskal_Wallis; ${ }^{\star}$ teste U de Mann-Whitney

Quanto aos valores médios de sobrecarga subjetiva o sexo masculino, viúvos, irmãos da pessoa doente, com o $1^{\circ}$ ciclo de formação literária e com um tempo de cuidado entre 7 a 10 anos evidenciam médias de sobrecarga subjetiva mais elevada $(2,36 ; 3,42 ; 3 ; 2,92$ e 3,04 respetivamente). Porém, só se verificaram diferenças estatisticamente significativas segundo o grau de escolaridade (KW=6,747; $\mathrm{p}=0,034)$, quanto mais elevada a escolaridade menor a perceção de sobrecarga. $\mathrm{Na}$ ajuda recebida os cuidadores com idades inferiores a 65 anos, os solteiros, os cônjuges, os que possuem o ensino superior, os empregados e os que cuidam entre 4 a 6 anos e mais de 10 anos parecem receber mais ajuda. Também neste caso nem o teste U d Mann-Whitney nem o de Kruskal-Wallis demonstraram evidência estatística para as diferenças entre os diversos grupos.

Os cuidadores do sexo feminino, com mais de 65 anos, os viúvos, os pais/mães, os que possuem o $1^{\circ}$ ciclo e com tempo de cuidado entre 4 a 6 anos são os que revelam médias mais elevadas de atitudes positivas. No entanto, a aplicação dos testes estatísticos não revelaram a existência de diferenças significativas entre os diferentes grupos.

Familiares do sexo feminino, com mais de 65 anos, viúvos, pais ou mães do paciente, que possuem o $1^{\circ} \mathrm{ciclo}$, empregados/desempregados e com um tempo de cuidado entre 1 e 3 anos, são os que evidenciam níveis mais elevados de criticismo. A aplicação dos testes estatísticos não revelaram significâncias estatísticas nos diferentes grupos estudados. 


\section{DISCUSSÃO DOS RESULTADOS}

A maioria dos familiares de referência/cuidadores do doente mental com esquizofrenia são do género feminino (54,3\%) e são progenitores (60\%). Resultados semelhantes foram encontrados em três estudos realizados em Portugal (Guedes, 2008; Pinho e Pereira, 2015; Xavier et al., 2002).

Durante milhares de anos a prática dos cuidados correntes, os cuidados que suportam a vida, ligou-se fundamentalmente à atividade dos diversos papeis que foram sendo atribuídos à mulher ao longo do desenvolvimento das sociedades, fruto da divisão social do trabalho. Assim, desde os tempos mais recuados da história da humanidade até ao fim da Idade Média, passando pelo período da Revolução Industrial, da descoberta da Célula e mais tarde da Bactéria tem havido uma identificação da prática dos cuidados com a mulher, papel modelado em torno da fecundidade e de todas as formas simbólicas que gera. Também, na atualidade, a prática dos cuidados quer cultural quer socialmente tem sido assumida pela mulher, a quem tem cabido a responsabilidade de cuidar das crianças, dos velhos e dos doentes (Pimentel, Mata \& Pereira, 2011). Nesta linha de análise, Mata \& Veja Rodrigues (2012) aludem à maior prevalência de enfermidades crónicas e incapacitantes associadas ao envelhecimento e deste modo o maior número de dependências e a necessária presença de um cuidador. Esta função, segundo as autoras, tem sido habitualmente assumida pela família, resultados em concordância com os do nosso estudo e corroborados pelo INE (2011) e Tobio, Agulló, Gómez \& Martín (2010). Também Afonso (2010); Pereira e Almeida (1999) e Torrey (2006) salientam a dependência familiar e a necessidade de ajuda constante destes doentes mentais em inúmeras atividades da sua vida diária.

São vários os estudos que indicam que a presença de um doente mental na família provoca uma elevada sobrecarga para os seus elementos (Brito, 2006; Borba et al., 2008; Guedes 2008; Pinho \& Pereira, 2015; Xavier et al., 2002). Analisando os resultados obtidos verificou-se a existência de sobrecarga objetiva $(1,99)$ e subjetiva $(2,35)$ na amostra em estudo, embora os valores não sejam demasiado elevados, sendo a sobrecarga subjetiva superior. Nos três estudos realizados em Portugal (Guedes 2008; Pinho \& Pereira, 2015; Xavier et al., 2002) obtiveram-se resultados semelhantes mas somente no estudo de Xavier et al., (2002), ao contrário dos outros dois, a sobrecarga subjetiva foi superior.
No que respeita à sobrecarga objetiva observaram-se pontuações mais elevadas nos problemas com o sono $(2,31)$ e negligênciar outros familiares $(2,28)$, ao contrário do observado no estudo de Xavier et al., (2002) com valores mais elevados para a dificuldade em fazer férias $(2,31)$ e limitação dos períodos de lazer $(2,02)$. $\mathrm{O}$ índice global de sobrecarga subjetiva foi o mais elevado do FPQ com pontuações superiores no desapontamento pela evolução do doente $(2,89)$; responsabilização do doente $(2,57)$ e preocupação com o futuro dos familiares (2,54). Também no estudo de Xavier et al., (2002) o desapontamento pela evolução do doente foi o item com pontuação mais elevada $(2,82)$, seguindose a responsabilização do doente $(2,58)$ e a preocupação com o futuro dos familiares $(2,26)$. Observaram-se diferenças com significado estatístico entre o grau de escolaridade e os valores médios da subescala de sobrecarga subjetiva ( $\mathrm{KW}=6,747 ; \mathrm{p}=0,034)$. Xavier et al., (2002) chegaram a idêntica conclusão. Estes resultados parecem evidenciar que à medida que a escolaridade aumenta, diminui a sobrecarga subjetiva o que nos leva a pensar que a resolução de problemas parece mais desenvolvida nos familiares com mais escolaridade. Porventura, maior facilidade no acesso à informação e por consequência maior capacidade para lidarem com as limitações decorrentes da doença mental e maior capacidade para gerir as emoções e sentimentos face ao problema.

Relativamente à ajuda recebida a média do índice global foi de 2,33 com as pontuações mais elevadas nos itens: ajuda por parte dos enfermeiros $(2,97)$; informação por profissionais de saúde $(2,58)$ e emergência/ ajuda de profissionais de saúde $(2,53)$. Tendo em conta que a pontuação mais elevada se revelou na ajuda por parte dos enfermeiros podemos inferir que os familiares se sentem apoiados por estes profissionais. Já a ajuda por parte de outras entidades obteve a pontuação mais baixa $(1,51)$ refletindo a escassez de recursos na comunidade para apoiar estas famílias. Além disso, a ajuda de familiares e amigos foi o segundo item com uma média mais baixa $(2,30)$ o que reflete a dificuldade sentida relativamente a este tão importante e cada vez menos disponível apoio de outros familiares ou de amigos, numa rede de suporte mais alargada.

As atitudes positivas obtiveram uma média de 2,34 sendo o item mais pontuado o elogio das qualidades do doente $(2,44)$. Também neste item, o estudo de Xavier et al., (2002), revelou resultado inverso ao aqui verificado. 
O departamento onde decorreu o estudo criou recentemente uma rede de suporte à família no pós internamento, com equipas comunitárias e serviços para proporcionarem apoio no cuidado e informação/educação em saúde, o que poderá ajudar a compreender os resultados observados nesta subescala. Com esta rede de suporte o sentimento de desalento pós alta pode ser, em parte, colmatado.

Relativamente ao criticismo a média da subescala foi de 1,71 , a mais baixa de todas as subescalas avaliadas. A vontade de se separar do doente $(1,43)$ foi um dos itens de menor pontuação sugerindo que apesar da sobrecarga identificada os familiares não pretendem deixar de cuidar dos seus doentes.

Igualmente no estudo de Xavier et al., (2002) este foi o item menos pontuado.

Quanto ao tempo de contacto com o doente, atendendo aos resultados obtidos, questionamo-nos se o número de anos de convivência com o doente resultará num aumento da sobrecarga, sendo necessários mais estudos para podermos chegar a alguma conclusão.

\section{CONCLUSÕES}

Como principais resultados destacamos a sobrecarga familiar objetiva e subjetiva dos familiares de doentes com esquizofrenia enquanto realidade à qual os profissionais de saúde não podem ficar indiferentes. Estes profissionais devem fazer parte integrante das intervenções educativas ao nível dos cuidados de saúde primários, se possível no meio envolvente do doente, nomeadamente, seguindo a recomendação da Resolução do Conselho de Ministros (2008), não apenas pelo conhecimento que detêm da família mas também pela relação terapêutica pré-estabelecida e pela continuidade dos cuidados após a alta hospitalar.

Assim, é importante dotar os profissionais de saúde em geral, e os enfermeiros em particularmente, de competências específicas para trabalhar com a problemática da doença mental grave.

A atuação do enfermeiro de cuidados de saúde primários, especialista de saúde mental e psiquiátrica, no planeamento das intervenções ao doente mental e suas famílias, per si ou integrado em equipas multidisciplinares, será uma mais-valia não só no que diz respeito à avaliação do contexto familiar, como também na participação da reabilitação psicossocial e continuidade de cuidados.

\section{IMPLICAÇÕES PARA A PRÁTICA CLINICA}

Este estudo permite perceber que a sobrecarga, sobretudo a subjetiva, bem como a necessidade de apoio por parte dos familiares de doentes com esquizofrenia, pelo que é importante delinear intervenções de enfermagem que ajudem a minimizar o problema.

Sugere-se a participação ativa do enfermeiro, especialista em saúde mental e psiquiátrica, na capacitação dos familiares e nas intervenções que reduzam a sobrecarga na família e comunidade.

\section{REFERÊNCIAS BIBLIOGRÁFICAS}

Afonso, P. (2010). Esquizofrenia - para além dos mitos, descobrir a doença. Cascais: Princípia.

Almeida, K. S., Dimenstein, M., e Severo, A. K. (2010). Empoderamento e atenção psicossocial: Notas sobre uma associação de saúde mental. Interface-Comunicação, Saúde, Educação, 14(34).

American Psychiatric Association. (2014). DSM 5 - Diagnostic and Statistical Manual of Mental Disorders (5a ed.). Washington: American Psychiatric Association.

Borba, L. O., Schwartz, E., \& Kantorski, L. P. (2008). A sobrecarga da família que convive com a realidade do transtorno mental. Acta Paulista de Enfermagem, 21(4), 588-594.

Caldas de Almeida, J. M., Xavier, M., Cardoso, G., Pereira, M. G., Gusmão, R., Corrêa, B., ... e Silva, J. (2013). Estudo epidemiológico nacional de saúde mental - $1^{\circ}$ relatório. Lisboa: Faculdade de Ciências Médicas da Universidade Nova de Lisboa.

Carvalho, J. C. (2012). Diagnósticos e intervenções de enfermagem centradas no processo familiar da pessoa com esquizofrenia. Revista Portuguesa de Enfermagem de Saúde Mental (8), 52-57.

Gomes, R. M. T. (2015). Psicoeducação unifamiliar em famílias de pessoas com doença mental grave (Tese de Doutoramento) Setúbal: Instituto Politécnico de Setúbal, Escola Superior de Saúde.

Gonçalves-Pereira, M., Xavier, M., Neves, A., Barahona-Correa, B., e Fadden, G. (2006). Intervenções familiares na esquizofrenia: Dos aspectos teóricos à situação em Portugal. Acta Médica Portuguesa, 19(1), 1-8. 
Guedes A. S. M. (2008). Avaliação do impacto da perturbação mental na família, e implementação de um programa psicoeducacional (Dissertação de Mestrado). Porto: Faculdade de Medicina da Universidade do Porto.

Instituto Nacional de Estatística. (2011). CENSOS de 2011. Lisboa: Instituto Nacional de Estatística.

Lucksted, A., McFarlane, W., Downing, D., \& Dixon, L. (2012). Recent developments in family psychoeducation as an evidence-based practice. Journal of Marital and Family Therapy, 38(1), 101-121. doi: 10.1111/ j.1752-0606.2011.00256

Mata, M. A., \& Vega Rodrigues, M. T. (2012). El cuidado no remunerado de mayores dependientes en el noreste de Portugal. Prismasocial (8), 333-357.

Observatório Português dos Sistemas de Saúde. (2015). Relatório de Primavera. Acesso aos cuidados de saúde: Um direito em risco?. Lisboa: Observatório Português dos Sistemas de Saúde.

Pereira, M. G., e Almeida, J. M. (1999). Repercussões da doença mental na família: Um estudo de familiares de doentes psicóticos. Acta Médica Portuguesa, 12(46), 161-168.

Pfammatter, M., Andres, K., \& Brenner, H. (2012). Manual de psicoeducação e gestão da doença na esquizofrenia. s/l: Edição Encontrar-se.

Pharoah, F., Mari, J., Rathbone, J., \& Wong, W. (2010). Family intervention for schizophrenia. Cochrane Database of Systematic Reviews, 12. doi: 10.1002/14651858. CD000088

Pimentel M. H., Mata, M. A. P., \& Pereira, F.A. (2011). The construction of social and professional identity of a feminine profession: Nursing. Prismasocial (7),1-23.

Pinho, L. M., e Pereira, A. M. (2015). Intervenção familiar na esquizofrenia: Redução da sobrecarga e emoção expressa. Revista Portuguesa de Enfermagem de Saúde Mental (14), 15-23.

Resolução do Conselho de Ministros. (2008). Plano Nacional de Saúde Mental 2007-2016. Diário da República, 1. a série, 47, 6 de Março, 1395-1409.
Silva, R. C. B. (2006). Esquizofrenia: Uma revisão. Psicologia USP, 17(4), 263-285.

Stephanie, T. (2012). The spirit level: Why more equal societies almost always do better. Leadership and Policy in Schools, 11(1), 129-134. doi: $10.1080 / 15700763.2011 .577928$

Tenório de Albuquerque, E. P., Oliveira Cintra, A. M., e Bandeira M. (2010). Sobrecarga de familiares de pacientes psiquiátricos: Comparação entre diferentes tipos de cuidadores. Jornal Brasileiro de Psiquiatria, 59(4), 308-316.

Tobío, C., Agulló, M. S. T., Gómez, M. V., \& Martín, M. T. P. (2010). El cuidado de las personas; Un reto para el siglo XXI. Barcelona: Fundación La Caixa.

Tomás, M. (2013). As intervenções do enfermeiro especialista em enfermagem de saúde mental e psiquiatria para os familiares das pessoas com o diagnóstico médico de esquizofrenia (Tese de Doutoramento). Setúbal: Instituto Politécnico de Setúbal, Escola Superior de Saúde.

Torrey, E. F. (2006). Surviving schizophrenia: A manual for families's, patients, and providers. New York: Harper Collins Publishers.

Xavier, M., Pereira, M. G., Corrêa, B. B., e Caldas de Almeida, J. M. (2002). Questionário de problemas familiares: Desenvolvimento da versão portuguesa de um instrumento de avaliação de sobrecarga familiar. Psicologia, Saúde e Doenças, 3(2), 165-177.

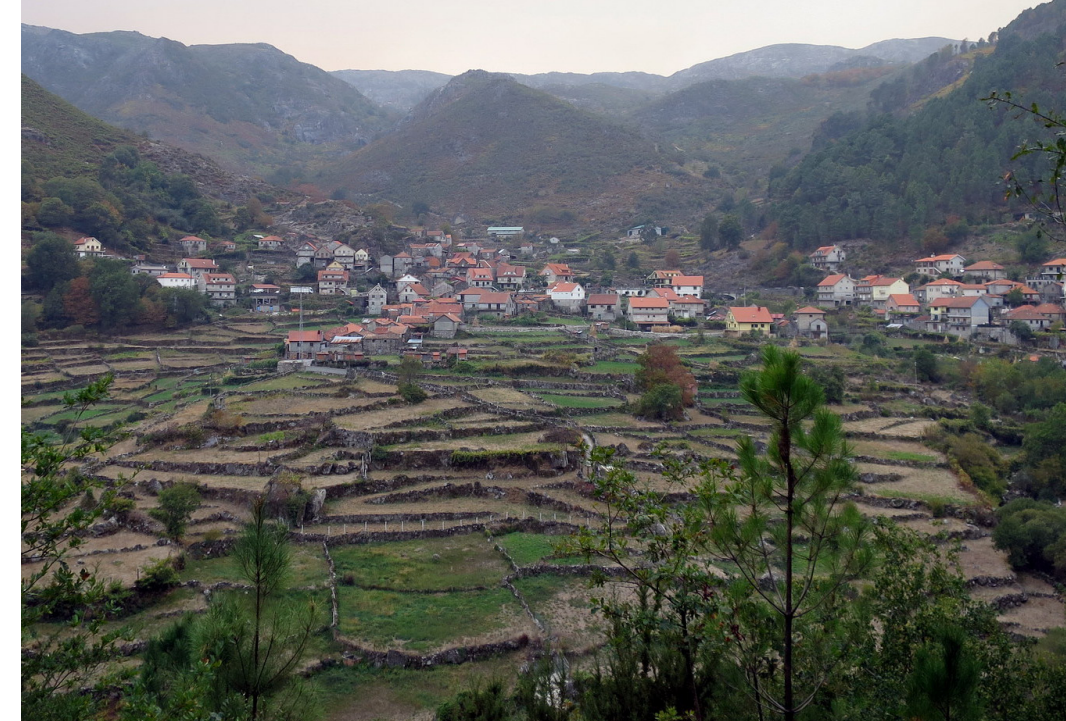

